\title{
Laboreal
}

Volume $2 \mathrm{~N}^{\circ} 1$ | 2006

Varia

\section{Entre lo local y lo global : proceso de regulación para la preservación de la salud en el trabajo}

Entre o local e o global : processos de regulação para a preservação da saúde no trabalho

Entre le local et le global : processus de régulation pour la préservation de la santé au travail

Between the local and the global : regulation process to health at work preservation

\section{Carla Barros-Duarte}

\section{(2) OpenEdition}

\section{Journals}

Edición electrónica

URL: http://journals.openedition.org/laboreal/13766

DOI: $10.4000 /$ laboreal. 13766

ISSN: 1646-5237

\section{Editor}

Universidade do Porto

Referencia electrónica

Carla Barros-Duarte, « Entre lo local y lo global : proceso de regulación para la preservación de la salud en el trabajo », Laboreal [En línea], Volume $2 N^{0} 1$ | 2006, Publicado el 01 julio 2006, consultado el 24 septiembre 2020. URL : http://journals.openedition.org/laboreal/13766 ; DOI : https://doi.org/ 10.4000/laboreal.13766

Este documento fue generado automáticamente el 24 septiembre 2020

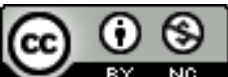

Laboreal está licenciado com uma Licença Creative Commons - Atribuição-NãoComercial 4.0 Internacional. 


\section{Entre lo local y lo global : proceso de regulación para la preservación de la salud en el trabajo}

Entre o local e o global : processos de regulação para a preservação da saúde no trabalho

Entre le local et le global : processus de régulation pour la préservation de la santé au travail

Between the local and the global : regulation process to health at work

preservation

Carla Barros-Duarte

\section{REFERENCIA}

Barros-Duarte, C. (2004). Entre o local e o global : processos de regulação para a preservação da saúde no trabalho. Tese de Doutoramento. Faculdade de Psicologia e de Ciências da Educação da Universidade do Porto, Porto.

\section{NOTA DEL EDITOR}

Manuscrito recibido en : abril/2006

Aceptado tras peritage en : junio/2006

\section{Introducción}

1 El punto de partida de este trabajo ha sido la constatación, a pesar de que estudios científicos desarrollados en el dominio de la salud en el trabajo han venido a demostrar 
la influencia del trabajo y de las condiciones de trabajo en la salud y en el bienestar de los trabajadores, que se asiste aún a pocos cambios en la concretización de una política global e integradora de la dimensión del trabajo en el planteamiento de la salud. De hecho, la poca visibilidad social de los datos estadísticos es muchas veces reforzada por la ausencia de voluntad de los mismos trabajadores en hablar de su trabajo y de sus condiciones de trabajo, optando, así, por una discreción social Canguilhem (2002), y, consecuentemente, para la ocultación de los efectos del trabajo en la salud.

2 En realidad, las relaciones entre la salud y el trabajo se revisten de una gran complejidad, "no son ni unívocas ni instantáneas" (Gollac \& Volkoff, 2000, p. 23) se establecen relaciones múltiplas y recíprocas que, superando los tradicionales diagnósticos de enfermedad, se traducen, también, en un conjunto de fenómenos y estados no patológicos pero reveladores de sufrimiento (Marquié, 1999) y que siguen, aún, poco visibles

3 Ha sido la confrontación con esta aparente paradoja que ha incentivado el proceso de investigación desarrollado, teniendo como principal objetivo comprender la ausencia de visibilidad de los efectos del trabajo en la salud recorriendo designadamente, a partir del marco teórico de la psicología del trabajo y de la ergonomía de la actividad, al estudio de los procesos de regulación asumidos por los trabajadores.

En verdad, en este trabajo, teniendo como contexto de investigación el sector de la Industria Textil y del Vestuario (ITV), han sido realizados un conjunto de estudios situados en tres niveles de análisis que, entre lo global y lo local, han permitido dar otra visibilidad a la cuestión de la salud en el trabajo :

- A nivel sectorial ha sido desarrollado un estudio del tipo epidemiológico a partir de la Encuesta SET - Encuesta Salud, Edad y Trabajo ;

- A nivel de la empresa ha sido realizado un estudio de caso en una de las empresas participantes en la Encuesta SET donde se ha desarrollado una investigación-acción en un colectivo de trabajadores ;

5 A nivel local ha sido desarrollado un estudio en el seno de la comunidad, fuertemente dominada por la ITV, donde se localiza la empresa estudiada: aquí, se ha intentado analizar el papel que los diferentes grupos de protagonistas en el área de la salud y de la salud pública tienen en el registro y diagnóstico de los efectos del trabajo en el estado de salud.

\section{Salud y trabajo : relaciones y evoluciones}

6 La investigación desarrollada tuvo subyacente los principales resultados de los estudios de los procesos de envejecimiento en y por el trabajo (Teiger, 1989 ; Marquié, Paumès, \& Volkoff, 1995 ; Derriennic, Touranchet \& Volkoff, 1996; Volkoff, Molinié \& Jolivet, 2000 ; Cassou et al., 2001) intentando realzar la complejidad del análisis de las relaciones entre el trabajo y la salud, designadamente al nivel del impacto de las condiciones de trabajo. De hecho, la diversidad y la variabilidad que caracterizan las relaciones entre salud y trabajo envuelven la comprensión de un conjunto de factores que interactúan mutuamente y a diferentes niveles en los varios momentos de la vida del individuo solicitando, así, un planteamiento global y plural.

7 Se sabe que, en realidad, las consecuencias del trabajo en el estado psicofisiológico no tiene, habitualmente, una expresión inmediata y lineal y, aunque acompañen todo el 
ciclo de vida del individuo, se manifiestan frecuentemente de forma diferida y singular estableciéndose relaciones diversas y recíprocas con el trabajo (Gollac \& Volkoff, 2000), que no son vivenciadas de la misma forma por todos los trabajadores

8 La Encuesta SET - Salud, Edad y Trabajo - cuya lógica de base se encuadra en los estudios de tipo epidemiológico, ha sido construido a partir de las aportaciones de las encuestas ESTEV $(1990,1995)$ y VISAT $(1996,2001)$ que, fuera de Portugal, ya han demostrado sus potencialidades.

El objetivo principal de este tipo de instrumento es el de comprender como y en que medida las condiciones de trabajo actuales y pasadas influencian, favorable o desfavorablemente, la calidad del éxito de envejecimiento y la evolución del estado de salud.

En esta perspectiva, la Encuesta SET ha intentado reconstruir el recorrido y la historia profesional del trabajador, así como la evolución de las condiciones de su salud. De hecho, la Encuesta SET, ha intentado superar los objetivos de los primeros modelos de epidemiología profesional, basados en el estudio de la relación directa y unívoca entre una causa y una patología específica, para modelos de epidemiología más recientes en que se intenta integrar otros factores designadamente las características profesionales, las condiciones de trabajo, los factores extra-profesionales y las condiciones de vida, abarcando igualmente las quejas y los problemas dichos "infrapatológicos" en sus relaciones con el trabajo.

11 El análisis de las relaciones menos visibles entre la salud y el trabajo abre también una nueva perspectiva, una nueva forma de mirar para la salud en el trabajo, dirigida para un planteamiento centrado en el trabajador: la integración de la perspectiva del trabajador, traducida en las percepciones, en las quejas y en los sentimientos sobre su salud, posibilita un planteamiento más global y más dinámico.

12 El análisis de los resultados de la Encuesta SET ha contribuido para la identificación de los principales riesgos profesionales y de los problemas de salud más frecuentes en la ITV, subrayando los efectos no sólo de las características físicas del trabajo pero, también, del contenido y de la organización del trabajo, realzando la importancia de los pequeños problemas de salud asociados al trabajo.

13 Sin embargo, este estudio confirmó la necesidad de un análisis complementario, más específico de la problemática estudiada centrada en la comprensión de las estrategias y de los procesos de regulación desarrollados por los trabajadores en las situaciones concretas de trabajo lo que ha llevado al desarrollo de una investigación-acción junto de un colectivo de trabajadores.

\section{La preservación y construcción de la salud en el trabajo : procesos de regulación}

14 Teniendo como punto de partida los principios delineados por Faverge (1966), el estudio de los procesos de regulación de la salud en el trabajo suscita una reflexión que realza la especificidad y la singularidad del comportamiento del hombre en el trabajo designadamente en la búsqueda - no siempre fácil - de un equilibrio entre lo que es exigido por el cumplimiento de las normas de producción y lo que exige la preservación de la salud. 
15 En esta perspectiva, la intervención apela a un planteamiento más comprensivo que explicativo (Volkoff, 2002) : privilegia escuchar, entender y comprender la salud (Honoré, 2002) abriendo, así, camino hacia una nueva mirada, más centrada en la vivencia del trabajador y apoyado en una dimensión más existencialista de la salud (Canguilhem, 2002).

De hecho, el estudio de los procesos de regulación de la salud en el trabajo envuelve, la comprensión de las formas de actuar, de pensar y de sentir de los trabajadores (Lacomblez, 2004), también realza el debate de valores que atraviesa la gestión que cada uno hace de su vida de trabajo (Schwartz, 2000) : dar un significado y un sentido a lo que ocurre en el trabajo traduce la dimensión de cariz existencial que invade permanentemente el mundo del trabajo y que refleja frecuentemente lo que ocurre también en el mundo fuera del trabajo (Curie, 2002 ; Davezies, 2002).

17 El estudio desarrollado se ha concretado en una acción de formación realizada en una empresa de la ITV junto de un colectivo de doce trabajadores y tuvo como principal objetivo promover una reflexión sobre las consecuencias de las condiciones de trabajo en la salud y en el bienestar. La metodología utilizada se ha caracterizado por la combinación de entrevistas individuales $y$ de entrevistas colectivas que, progresivamente, han favorecido el compartir, la emergencia y la confrontación de diferentes representaciones sobre las relaciones entre la salud y el trabajo.

Los resultados han evidenciado cambios al nivel del discurso de los trabajadores, designadamente en las formas de pensar/reflexionar las relaciones entre la salud y el trabajo, revelando una progresiva sistematización colectiva de las condiciones de trabajo evidenciando, así, una otra formulación explicita en la toma de conciencia de las relaciones entre salud y trabajo.

19 El análisis de las estrategias de regulación desarrolladas por los trabajadores ha realzado aún una gran variedad de formas de regulación, adoptados por cada uno.

20 De hecho, los criterios referidos en la gestión de las restricciones de la actividad de trabajo muestran que, a pesar de traducirse sobretodo en la modificación y ajuste de las formas operativas - regulaciones operativas (Faverge, 1966) - a partir de las características antropométricas y morfológicas de cada uno, son también desarrolladas formas más peculiares de preservar su salud - regulaciones catacréticas (De Keyser, 1972) el trabajador modifica la finalidad de un determinado instrumento, acción o incluso pensamiento, de forma a adaptarse a la realidad concreta de trabajo preservando su salud y su bienestar. Las estrategias de regulación de la salud revelan, así, una forma muy propia de regular su trabajo de forma a disminuir las restricciones, recuperar el tiempo y alcanzar un equilibrio posible, revelando, también, la interferencia de valores personales decurrentes de una experiencia que no se limita al mundo laboral.

21 La confrontación y el debate colectivo centrado en lo real del trabajo han favorecido la posibilidad de decirse lo que hasta ese momento se vivía en silencio, de explicitar, de desarrollar, de mejor definir, de recurrir a las experiencias de la vida fuera del trabajo y de comparar formas de pensar/sentir, interpretaciones, estrategias y soluciones.

22 A lo largo del proceso de intervención el discurso de los trabajadores ha ido evolucionando: si en las primeras etapas, la gestión de la salud en el trabajo se caracterizaba por preocupaciones relacionadas, sobretodo, con la gestión del uso de si, 
gradualmente, han emergido un conjunto de valores que Yves Schwartz integra en el registro de los "valores sin dimensión "

distintos de los valores de mercado - que corresponden a los valores de la política, de la deliberación democrática, del derecho social, del derecho al trabajo (Schwartz, 2000). Y la situación colectiva del compartir con los pares y los investigadores parece haber tenido un papel decisivo para la formación de una red de apoyo integradora y de reflexión.

Todavía, esta intervención, por los conflictos por los que el proyecto de su alargamiento ha encontrado al nivel de la empresa, ha terminado por reorientar sus objetivos en un campo de intervención de la salud pública. Se ha intentado privilegiar, entonces, el interfaz con los centros de salud de la comunidad local, realzando más concretamente el papel de los médicos de cabecera - responsables, también, por la evolución del estado de salud de estos trabajadores.

\section{La organización de la salud : intervenir en la construcción de interfaces}

25 A partir de la experiencia conducida por Marc Andeol y Gilbert Igonet en la región de las "Bouches du Rhône" (Association pour la Prise en Charge des Maladies Eliminables, 2005 ; Le quotidien du Médecin, 2006), impulsada por Ivar Oddone (Oddone, 1999), se ha intentado analizar el interfaz entre la realidad previamente estudiada y los centros de salud de la comunidad local, más concretamente el papel de los médicos de cabecera.

El análisis de un conjunto de entrevistas individuales realizadas a 18 médicos de los centros de salud - ha revelado que, en las preocupaciones con los cuidados globales de salud y de salud pública, persiste, aún, una insuficiente integración de la influencia determinante que el trabajo puede tener en la salud y en el bienestar - en una región en que es elevada la frecuencia de enfermedades profesionales típicas de los trabajadores de la ITV, como la sordera profesional y la bisinosis ( $54 \%$ y $28 \%$, respectivamente, de las enfermedades profesionales reconocidas en aquella comunidad local).

De hecho, la mayor parte de los médicos afirma desconocer el tipo de actividad de trabajo desarrollada en la ITV así como las condiciones de trabajo más típicas en las empresas del sector, a pesar de que la mayoría de sus usuarios ( $80 \%)$ trabaja o ya han trabajado en este sector.

La aparente incoherencia entre las enfermedades profesionales reconocidas y el contenido del discurso de los médicos de cabecera constituye un factor singular en términos de intervención: por un lado, "la gran mayoría de los médicos no posee ningún conocimiento del trabajo industrial (en sentido lato, es decir, del trabajo en la sociedad industrial) y no existe ningún incentivo profesional o financiero" para tal (Thébaud-Mony, 1991, p. 71), por otro lado, el aislamiento profesional en el cual acaban por asumir sus funciones es flagrante así como la ausencia de cualquier apoyo al nivel de los conocimientos e informaciones sobre la realidad de trabajo. Y, consecuentemente, la prevención desarrollada se aleja, cada vez más, del lugar de trabajo, privilegiando el control de los factores de riesgo subyacentes a los comportamientos individuales y la promoción de " estilos de vida sanos" (Ministerio de la Salud, 2004), dependientes, aparentemente, de la buena voluntad de los trabajadores. 


\section{Perspectivas y retos}

Las cuestiones relativas a la salud en el trabajo apelan a una intervención a varios niveles que supera, ciertamente, el nivel de la empresa y envuelve diferentes actores que, al nivel local, pueden interferir en la preservación y construcción de la salud y del bienestar.

Sin embargo, las acciones existentes se muestran aún insuficientes poniendo en cuestión los actuales mecanismos de regulación institucionales que, muy dependientes del poder central, acaban por llevar al aislamiento los organismos locales, dificultando el análisis de las necesidades más específicas de la comunidad.

1 La implementación de redes y estructuras de apoyo que favorezcan la construcción de capacidades individuales y colectivas de debate y de acción (Davezies, 2002) en el dominio de la salud necesita, pues, de otra forma de perspectivar la organización de la salud al nivel local que pasa, sobretodo, por el desarrollo de procesos concretos de coordinación entre los diferentes grupos de protagonistas que pueden interferir en la preservación y construcción de un bien común que es la salud.

Los resultados de esta investigación evidenciaron la necesidad del refuerzo y/o de la creación de estructuras y redes de apoyo, susceptibles de asumir mejor la función reguladora de una política de la salud que dejaría de olvidar que la mayor parte de los ciudadanos ejerce una actividad profesional.

\section{BIBLIOGRAFÍA}

Association pour la Prise en Charge des Maladies Eliminables (2005). Rapport d'Activité 2003-2006. Évaluation interne de l'utilisation des Fonds d'Aide à la Qualité des Soins de Ville (FAQAV). Bouchesdu-Rhône : APCME.

Canguilhem, G. (2002). Ecrits sur la médecine. Paris : Éditions du Seuil. Cassou, B., Buisset, C., Brugère, D., Davezies, P., Derriennic, F., Desplanques, G., Laville, A., Marquié, J-C., Touranchet, A., \& Volkoff, S. (2001). Travail, Santé, Vieillissement : relations et évolutions. Toulouse : Éditions Octarès.

Curie, J. (2002). Dis-moi, Docteur, c'est grave ? Manuscrito não publicado.

Davezies, P. (2002). Bilan et défis. Prévention-Sécurité, 61, 30-38.

De Keyser, V. (1972). Fiabilité et expérience. In CECA, Études de Physiologie et de Psychologie du Travail, no 7, Fiabilité et Sécurité Eléments pour une ergonomie des systèmes en milieu industriel (pp. 77-137). Luxembourg : Diffusion des Connaissances - Commission des Communautés Européennes.

Derriennic, F., Touranchet, A. \& Volkoff, S. (1996). Age, travail, santé. Paris : Les Editions INSERM.

Faverge, J.M. (1966). L'analyse du travail en terme de régulation. In : J.M. Faverge, M. Olivier, J. Delahaut, P. Stephaneck \& J.C. Falmagne. (Eds.), L'ergonomie des processus industriels (pp. 33-60). Bruxelles : Editions de l'Institut de Sociologie, Université de Bruxelles. 
Gollac, M. \& Volkoff, S. (2000). Les conditions de travail. Paris : Editions La Découverte.

Honoré, B. (2002). A Saúde em Projecto. (I. d’Espiney, Trad.). Loures : Lusociência.

Lacomblez, M. (2004, Janeiro) De Faverge à Vergnaud : entre variabilité et invariants dans le développement de l'activité. Communication présentée au colloque international ARDECO : Les processus de conceptualisation en débat : hommage à Gérard Vergnaud. Paris, France.

Le quotidien du Médecin (2006). Le quotidien du Médecin [version électronique]. Retiré le 7 février 2006, de http://www.quotimed.com/journal/index.cfm? fuseaction=viewarticle\&DartIdx $=22113243 \mathrm{k}$.

Marquié, J-C. (1999). Quelques composantes psychiques et cognitives de la relation âge, travail, santé. Colloque Santé, Travail, Vieillissement : Relations et Évolutions. Paris : CREAPT.

Marquié, J-C., Paumès, D. \& Volkoff, S. (1995). Le travail au fil de l'âge.

Toulouse : Éditions Octarès.

Ministério da Saúde (2004). Programa nacional de intervenção integrada sobre determinantes da saúde relacionados com os estilos de vida [version électronique]. Retiré le 3 février 2005, de http://www.dgsaude.pt

Oddone, I. (1999). Psicologia dell'organizzazione della salute. Psicología della Salute, Fascicolo 1. Schwartz, Y. (2000). Le paradigme ergologique ou un métier de philosophe. Toulouse : Octarès.

Teiger, C. (1989). Le vieillissement différentiel par et dans le travail : un vieux problème dans un contexte récent. Le Travail Humain, 52, 1, 21-56.

Thébaud-Mony, A. (1991). La reconnaissance des maladies professionnelles : acteurs et logiques sociales. Paris : La Documentation Française.

Volkoff, S. (2002). Des comptes à rendre : usages des analyses quantitatives en santé au travail pour l'ergonomie. Noisy-le-Grand : Centre d'Etudes de l'Emploi.

Volkoff, S., Molinié, A-F., \& Jolivet, A. (2000). Efficaces à tout âge ? Vieillissement démographique et activités de travail. Paris : Dossier du Centre d’Études de l’Emploi oํ 16.

\section{AUTOR}

\section{CARLA BARROS-DUARTE}

Universidade Fernando Pessoa Praça 9 de Abril, nº 349, 4249-004 Porto cbarros@ufp.pt 